\title{
LAS SECRETARIAS DE LOS CONSEJOS, LAS DE ESTADO Y DEL DESPACHO Y SUS OFICIALES DURANTE LA PRIMERA MITAD DEL SIGLO XVII.*
}

\author{
por \\ CONCEPCION DE CASTRO \\ Universidad Complutense de Madrid.
}

RESUMEN: Apenas se trata aqui de los antiguos Consejos de la Monarquía Hispánica ni de su supervivencia en el siglo XVIII; interesa más, en cambio, la nueva figura de los Secretarios de Estado y del Despacbo, precedente de los ministros contemporá neos. L Lama la atención, durante el largo reinado de Felipe $V$, la frecuencia con la que esos Secretarios llegaban al poder como culminación de una larga vida administrativa. De abi que el articulo se centre en sus Secretarias u oficinas, asi como en las de los Consejos tradicionales cuyo modelo van a seguir las nuevas instituciones; en la camera de sus empleados $u$ "roficiales"y y en la procedencia social de los mismos; en la preparación profesional necesaria para acceder a tales plazas y en lo que podriamos llamar "familias administrativas» a que daba lugar el patronargo; finalmente, en la paulatina politización de los Secretarios o ministros y la de sus oficiales a partir de la muerte de Carvajaly la caida de Ensenada. Por otro lado, se aportan algunos ejemplos de Los conflictos surgidos al principio entre las antiguas instituciones y las nuevas. Resultaban inevitables a todos los niveles, dada la pérdida de poder de los Consejos bajo la dinastia de los Borbones y, con éllo, la disminución de sus Secretarias frente al auge de las nuevas del Despacho.

Palabras Clave: Consejos y sus Secretarias. Secretarios de Estado y del Despacho y sus Secretarias (Ministerios). «Oficiales" (empleados públicos). Patronazgo. Experiencia administrativa u *oficio de papelesw, Regalismo, antijesuitismo y politica ilustrada.

\footnotetext{
* Para no hacer farragoso este artículo, he pasado por alto etapas muy breves en las reformas de Felipe V. Me refiero, por ejemplo, a la Veeduría General e Intendencia de Hacienda de 1714, en lugar del ministerio correspondiente; a la división en sólo tres ministerios, de 1717, $o$ a las interesantes pero pronto malogradas reformas de Macanaz. También he llamado «ministerios» a su precedente, las Secretarias de Estado y del Despacho, con el objeto de hacer más sencilla la exposición y evitar confusiones con las Secretarías de los Consejos.
} 
ABSTRACT: The former Councils of the Spanish Monarcby and their survival in the 18th century are barely touched upon bere. Of greater interest, bowever, are the new posts of Personal Secretary and Secretary of State and Office, the forerunner to contemporary ministers. Notewortby during the long reign of Philip $V$ was the frequency with which the arrival in power of these Secretaries was the aulmination of a long life of administrative duty. The article thus focuses on their Secretariats or offices, and on those of the traditional Councils, the model of which was to be followed by the new institutions; on the career pursued by their employees or "rofficials" and on their social background; on the career training needed to gain access to these new posts and on what we might call the «administrative families" arising from patronage; and finally, on the gradual politicising of the Secretaries or ministers and that of their officials following the death of Carvajal and the fall of Ensenada. Moreover, some examples are given of the conflicts initially arising between the former and new institutions. These were inevitable at all levels in view of the loss of power of the Councils under the Bourbons and, in tandem, the diminished authority of the Councils Secretaries compared with the rise in that of the new Personal Secretaries.

KEY wORDS: Councils and their Secretaries. Secretaries of State and office and their Secretariats (Ministries). "Officials* (civil servants). Patronage. Administrative experience. Regalism, anti-Jesuitism and enlightenment policy.

Como es sabido, las reformas de Felipe $\mathrm{V}$ hicieron que los antiguos Consejos del sistema de gobierno de los Austrias perdieran poder. Sin que desaparecieran los Consejos, las nuevas Secretarías de Estado y del Despacho y sus Secretarios -los ministros- pasaron ahora al primer plano. Los Secretarios del Despacho iban a ser las figuras más cercanas al monarca, con quien despachaban personal y continuamente. Frente al poder tradicional de los Consejos como organismos colegiados, la función de los Secretarios del Despacho consistía en agilizar el gobierno y la administración; pero también en llevar a cabo un amplio programa de reformas y mejoras, lo cual les confería una importante iniciativa en sus respectivos sectores y recortaba el papel de los Consejos ${ }^{1}$.

Una de las manifestaciones del cambio fue la reducción en el número de consejeros, que en algunos Consejos había crecido en exceso a base de los miembros de «capa y espaday -con algunas plazas incluso enajenadas- y de otros supernumeratios ${ }^{2}$. También se redujo el personal de las Secretarías de esos Consejos tradicionales al

1 M. Artola: El Estado, en Enciclopedia de Historia de España, dirigida por èl mismo, T. II, Madrid, 1988, pp. 148-11149.

2 AHN, Est., Leg. 6379, 2.

Hijpanta, LIX/1, núm. 201 (1999) 193-215 
tiempo que se debilitó la prestigiosa figura de sus Secretarios, los cuales iban a seguir dirigiéndolas a lo largo del siglo XVIII. Lo propio de estas Secretarías eran las cuestiones administrativas o "manejo de los papeles", labor que hacía posible el funcionamiento de los Consejos. Aparte del Secretario al frente de cada una de ellas, estas oficinas estaban integradas por una serie de "oficiales» de plantilla o "del número" y por otros supernumerarios, con la esperanza, éstos últimos, de integrarse alguna vez entre los primeros; podía haber incluso simples oficiales «entretenidos» que, sin sueldo alguno, sobrevivían gtacias a determinadas gratificaciones. En las Secretarías de los Consejos solía haber también un archivero y, a veces, uno o más traductores ${ }^{3}$

Los miembros del primero y siempre más poderoso de los Consejos, el de Castilla, eran todos magistrados. Sus plazas no habían sido utilizadas, como en otros Consejos, para premiar servicios a la corona ni había recibido, por lo tanto, consejeros de capa y espada. Conservó hasta el final su propio gobernador, sin ser nunca presidido, como sucedieta en ottos Consejos, por el ministro del ramo correspondiente. El papel del Consejo de Castilla siguió siendo relevante en el siglo XVIII. Lo cual no quiere decir, sin embargo, que no quedara ahora sometido al poder ministerial; así lo exigía el nuevo sistema y sólo así podía sobrevivir el Consejo. Se manifiesta de forma muy clara en la actuación de una figura como la de sus fiscales, que destacan sobre sus homólogos en otros Consejos: Todos, o prácticamente todos, los asuntos tratados en el Consejo pasaban por sus manos; es más, eran ellos quienes redactaban los informes, o «respuestas fiscales», sobre los que trabajaba después el Consejo, con la capacidad que ésto les otorgaba para influir en la opinión de los consejeros. Por eso mismo eta preciso elegit para el Consejo fiscales que coincidieran con la línea programática de los ministros del Rey.

Una de las peculiaridades del Consejo de Castilla era la de no tener Secretaría alguna ni Secretario. El peso del despacho recaía aquí sobre sus seis escribanos de cámara, en primer lugar sobre los dos especializados en los asuntos consultivos y de gobierno - los denominados escribanos de cámara y de gobierno-. El status de éstos dos últimos era elevado, similar al de los Secretarios de otros Consejos; pero, a diferencia de éstos, no asistían a las deliberaciones del de Castilla, siempre secretas. Las oficinas de los escribanos del Consejo estaban en sus propios domicilios, y eran ellos quienes retribuían a los oficiales de las mismas. La Cámara de Castilla, organismo anejo al Consejo del mismo nombre y constituído por algunos de sus mismos consejeros, disponía, en cambio, de dos o tres Secretarías, dedicada cada una de ellas a los diferentes tipos de actividades que correspondían a la Cámara. Eran las de Gracia, Patronato y, ya en el siglo XVIII, la de Aragón. Había también una Secretaría del presidente o gobernador del Consejo.

3 Sobre las Secretarías y los Secretarios, en J.A. Escudero: Las Secretarios de Estado y del Despacbo, Madrid, 1969, 4 vols. y Los origenes del Consejo de ministros en España, Madrid, 1979, 2 vols.Cfr. también Gildas Bernard: Le Sécretariat d'Etat et le Conseil des Indes, Paris, 1972.

Hitpania, LIX/1, núm. 201 (1999) 193-215 
La carreta de los oficiales de las Secretarias de los Consejos no era en absoluto desdeñable, como no lo era tampoco su status. Pertenecían todos a familias hidalgas, por supuesto, entre las que abundaban las originarias del Norte, aunque a veces con varias generaciones de residencia madrileña. $Y$ muchos de los oficiales pertenecían desde su juventud a alguna de los Ordenes Militares más prestigiosas.

El ingreso en aquellas Secretarías se producía normalmente por abajo, en la última o en una de las últimas mesas de la oficina correspondiente y con sueldo aún escaso, de pocos miles de reales. A partir de ahí, se iba ascendiendo con el paso del tiempo y las vacantes hasta alcanzar el puesto de oficial mayor, con sólo el Secretario por encima. Después no quedaba más que ser Secretario y, pasando de la jefatura de una a otra Secretaria, legar a las primeras y de mayor rango, las del Consejo de Estado (Negociación de Italia o Negociación del Norte). El camino podía ser más rápido, llegando directamente a éstas dos últimas desde la correspondiente oficialia mayor. La importancia atribuida a los Secretarios del Consejo de Estado estaba basada en su capacidad de despacho directo con el monarca en el siglo XVI; después se habían interpuesto los validos y, a finales del XVII, al amparo de los vaivenes y sustituciones de validos, eta ya el Sectetario del Despacho Universal quien podía tratar directamente con el monarca. El del Despacho Universal habia surgido por la necesidad de que alguien se ocupara de las cuestiones puramente administrativas -de los papeles, en definitiva-mientras predominaron los validos ${ }^{4}$. Pero, dado el peso de la tradición en el Antiguo Régimen, llegar a Secretario del Consejo de Estado siguió significando después, durante el siglo XVIII, la culminación de una carrera administrativa. Los sueldos de estos Secretarios continuaron siendo, además, sustanciosos, superiores incluso a los de magistrados de tanto prestigio como los consejeros de Castilla.

La preparación necesaria para iniciar esa carrera administrativa en los Consejos no incluía el paso por la Universidad; pero sí el estudio de lenguas, con el latín incluido, y una cierta erudición y cultura. Requisito indispensable era, por supuesto, el de comprometerse a mantener secretos los negocios de las Secretarias. Ahora bien, la cualidad más apreciada era la experiencia profesional, lo que J.A. Escudero denomina experiencia en el «manejo de papeles». Ahí es donde entra de lleno el patronazgo; porque los Secretarios de los distintos Consejos introducían desde muy jóvenes a sus hijos y ottos familiares en las oficinas como simples meritorios, sin necesidad de plaza ni retribución alguna. Si se tiene en cuenta, además, que eran los mismos Secretarios quienes hacían las propuestas para cubrit las vacantes de sus respectivas oficinas, no quedan muchas dudas sobre quiénes llevaban la preferencia. De ahí que, con el tiempo, se llegaran a formar familias de administrativos y Secretarios; y que algunas de ellas se mantuvieran durante el reinado de Felipe V. Al mismo tiempo, una de las ideas dominantes, que se transmite al siglo XVIII, es la de que a cada indi-

4 Ver en las obras cits. de Escudero.

Ilipania, LIX/1, núm. 201 (1999) 193-215 
viduo le valen como un activo importante los méritos de otros familiares y ascendientes suyos al servicio de la corona. Estos méritos pueden haberse contraído en la mencionada administración, pero también en el ejército, la marina o la Casa Real; a veces se aducen incluso los de determinadas mujeres de la familia como camareras de la reina, por ejemplo. Lo normal cuando se "sirve» con acierto -afirma un informe anónimo de pricipios del XVIII- es pedit ascensos y gracias para sí mismo, para hijos y parientes. Resulta normal, por lo tanto, pedir cargos públicos como retribución a los servicios prestados por otros miembros de la familia ${ }^{5}$.

En cualquier caso, los Secretarios de los Consejos no introducían siempre a sus propios hijos en las oficinas; a veces, pedían para ellos puestos militares y, sobre todo, empleos palatinos que situaran al joven en el entorno de las personas reales ${ }^{6}$. Era entonces el turno de otros recomendados y favorecidos. Destacan entre ellos los pajes de personajes nobiliarios o del mundo político y cortesano, así como los de los mismos Secretarios. Estos pajes eran acogidos desde la adolescencia en el servicio de aquellas casas; allí se les enseñaba ante todo a escribir, aunque se les introducía también en el manejo de la correspondiente y más modesta secretaría. Cuando acompañaban las dotes personales, se podía empezar como paje y acabar en alguno de los más altos puestos del Estado. Es cierto que los nombres de muchos pajes se nos quedan perdidos por el camino; pero destacan otros que, como Juan Bautista de Orendain o Sebastián de la Cuadra, acabarían ocupando el ministerio de Estado.

El patronazgo actuaba, más que como forma de ascenso en la carrera, como medio de acceso a la misma. La familia, sus relaciones e influencias resultaban una base inapreciable de movilidad social, como mostraton $\mathrm{J}$. Fayard en su estudio sobre los consejeros de Castilla o J. Cruz en el suyo sobre los comerciantes y banqueros madrileños. Cruz ha demostrado también la importancia de las redes de solidaridad regional entre los grandes comerciantes y banqueros madrileños de orígen forastero. Era una solidaridad que, junto a la familiar, actuaba en todos los grupos y sectores de inmigración en la capital y que explica, entre otros muchos ejemplos, los apoyos que recibió Campomanes al llegar a Madrid en 1742. El enriquecimiento por sí solo no era suficiente; ni lo era tampoco la educación que aquel permitía si faltaban las relaciones adecuadas para acceder, al menos, a alguno de los caminos abiertos después a la constancia en el trabajo $y$ al mérito personal ${ }^{7}$.

No debía de ser fácil, desde luego, para las familias hidalgas de pocos recursos, encontrar acomodo para sus hijos sin dejarlos caer en la esfe-

\footnotetext{
5 Los ejemplos abundan en AHN, Est, Legs. 3496 y 3497.

6 Cfr. en AHN, Est, Leg. 3496, nº 48 .

7 Cfr. en J. FAYARD: Los miembros del Consejo de Castilla, Madrid, 1982; J. CruZ: Political Change and Cultural Persistence Among the Spanish Dominant Groups, 1750-1850. Cambridge University Press, 1996; C. de Castro: Campomanes. Estado y reformismo ilustrado, Madrid, 1996; y O. CHALINE: La France au XVIII siècle. 1715-1787, Paris, 1996, pp. 195 y ss.
} 
ra del trabajo manual y degradar con ello a toda la familia. En una sociedad tan desigual, el objetivo último de toda estrategia familiar era mantenerse o ascender en la escala social, ascender a veces para no caer $^{8}$. A parte de la carrera eclesiástica y de la militar, o de los empleos cortesanos y de los diplomáticos, las oportunidades profesionales eran escasas, como lo era la demanda existente. El paso por la Universidad abría el camino a las cátedras, a la práctica de la medicina o de la abogacía y a la magistratura. Sin necesidad de los años y el dinero que requería un título universitatio, se ofrecía ese oficio de los "papeles», es decir, el de la administración en sus diversos ámbitos: el militar, ya fuera en el ejército o en la marina, y el de las oficinas de la Hacienda Pública; el de la administración de las Ordenes Militares, o bien, aunque en menor medida, el de las distintas instituciones eclesiásticas., al que también tenían acceso los seglates; y, como queda dicho, el de las casas nobiliarias u otras que admitian a los mencionados pajes. La práctica adquirida en cualquiera de esos ámbitos constituía también un activo para acceder -cuando no se podía hacerlo directamente- al más importante y codiciado de todos, el de la administración civil en las Secretarías de los Consejos y, desde el siglo XVIII, también en las nuevas Secretarías del Despacho, precedente directo de los ministerios ?

El desdoblamiento de la del Despacho Universal en dos Secretarías, en septiembre de 1703 por primeta vez, matca un hito en el camino de las reformas políticas y administràtivas de Felipe V. Manuel Coloma y Escolano, ya por entonces segundo marqués de Canales, accedió a la Secretaría de Guerra; «todo lo demás» quedó en manos de Rivas, que venía desempeñando la Secretaría del Despacho Universal. De familia riojana, Canales procedía de la carrera de la magistratura, de donde había saltado a la diplomacia. Era hijo de un antiguo $\mathrm{Se}$ cretario de Estado de Felipe IV, y su hermano mayor lo había sido a su vez de Carlos II. La pérdida de Gibraltar en agosto de 1704 fue considerada como fracaso de Canales por Luis XIV, quien obligó a su nieto a prescindir de él y a volver a la gestión única de Rivas. Había sido un ensayo del joven monarca, quien iba a repetirlo muy pronto, en julio de 1705 , para perpetuarlo a partir de entonces.

Con la excepción de Canales, los nuevos Secretarios del Despacho - ya ministros, en realidad- proceden, durante este largo reinado, de las filas de la administtación que, durante bastante tiempo, serán necesariamente las filas de la antigua administración, la de las Secretarías de los Consejos. El Secretario del Despacho - o ministro- más importante durante aquellos años de guerra, el de Hacienda y Guerra, es José Grimaldo y Gutiérrez de Solórzano. Pertenecía a una de las ramas españolas de una muy antigua familia genovesa; su abuelo y su padre

\footnotetext{
8 M. Hernández: $A$ la sombra de la Corona. Poder local y oligarguia urbana. (Madrid,16061808) Madrid, 1995, p. 151.

9 Lo más frecuente en las familias de altos funcionarios parece haber sido destinar a la Universidad y a la larga carrera de la magistratura a los hijos menores, reservando para los primogénitos, junto al mayorazgo, la vía hacia el gobiemo del Estado.(M. Hernández, op. cit. pp. 183 y ss.).
}

Hípania, LIX/1, núm. 201 (1999) 193-215 
habían ascendido ya en la escala jerárquica de las Secretarías de los Consejos, y él mismo habia ingresado muy joven en una de las de Indias, la de Nueva España. Había pasado después a la de la Negociación del Norte, en el Consejo de Estado, y había destacado desde la llegada del primer Borbón. Al recibir el nombramiento de Secretario de Estado y del Despacho de Guerra y Hacienda contaba en su haber con 31 años de servicio a h monarquía. El Secretario, o ministro, de «todo lo demás», fue Pedro Cayetano Fernández del Campo Angulo y Velasco, segundo marqués de Mejorada, emparentado con una casa infanzona del valle de Tudela. Su padre había llegado a Secretario de las Negociaciones del Norte y de Italia y, como tantos otros, había iniciado a su hijo en la profesión desde temprano; ya con Felipe V, éste último había alcanzado una de las Secretarías del Consejo de Estado. Son carreras similares a la de su predecesor, el último Secretatio del Despacho Universal, Antonio de Ubilla y Medina ${ }^{10}$.

La misma tónica va a seguir predominando cuando, desde 1714, esos dos primeros ministerios se subdividan definitivamente en cinco. (Estado, Hacienda, Guerra, Justicia y Marina e Indias). Asi, Manuel de Vadillo y Velasco había iniciado su carreta profesional como oficial en la Secretaría de Estado para la Negociación de Italin, llegando con el tiempo a disfrutar las Secretarias de Ordenes, del Consejo de Italia y del Real Patronato de la Cámara de Castilla; después, fue ministro de Justicia entre 1714 y 1724, con José Grimaldo en Estado, que desde el primer momento se configura como el ministerio principal Miguel Fernández Durán, ministro de la Guerra también con Grimaldo, había empezado como oficial entretenido en la Secretaría del Consejo de Guerra y, mientras ascendía por la antigua escala administrativa, habia sido admitido como caballeto de Calatrava. El ministro de Matina e Indias, también en 1714, Bernardo Tinajero de la Escalera, había ido subiendo en una de las Secretarías del Consejo de Indias hasta llegar a Secretario de ese Consejo en 1705. El único ministro surgido de las filas de la magistratura durante aquella etapa fue José Rodrigo Villalpando, que llegó a Justicia tras haber sido fiscal del Consejo de Castilla, aunque también había desempeñado alguna misión diplomática durante la Guerra de Sucesión.

Una figura interesante es la de Juan Bautista de Orendain, y no sólo porque trabajara en una y otra administración, la antigua y la nueva, antes de llegar a encabezar el ministerio de Estado. Habia nacido en Azpeitia, aunque en el seno de una familia de orígen navarto. Había comenzado su camino profesional como paje de Grimaldo, que siguió siendo después su protector. De ahí que entrara primero en una Secretaría del Consejo de Indias y, tras ascender en ese escalafón, pasaría después al ministerio de Estado, donde llegó hasta la primera mesa ${ }^{11}$. Al abdicar Felipe $\mathrm{V}$ en su hijo Luis I, en enero de 1724 , y permanecer Grimaldo junto al rey padre, Orendain ocupó el primer lugar en el ministerio preparado al joven Luis. En el

10 Cfr. en A. y A. GARCIA CARRAFFA: Enciclopedia beráldica y genealógica hispano-americana, tomos 22 y 78; en J.A. Escudero, op. cit; y en J. Martinez Cardós: Primera Secretaría de Estada. Ministerio de Estado, Disposiciones orgánicar, Madrid, 1972.

11 El curriculum de Orendain, según aparece en AHN, Est, Leg. 3497, es, en realidad, más complicado; pero conviene resumirlo pot tazones de legibilidad. 
mismo figuraban asimismo otros fieles a Grimaldo, como Rodrigo Villalpando y Baltasar Patiño. Pero la pieza clave para mantener el poder en las mismas manos era Orendain, siempre al servicio de su señor, el marqués de Grimaldo. Al volver éste último con Felipe V, a finales del mismo año de 1724, Orendain ocupó el ministerio de Hacienda que, con la breve interrupción del barón de Riperdá, mantuvo hasta el retiro de su protector a finales de 1726. Pasó entonces a sucederle en Estado, ministerio que conservó otros ocho años.

La desaparición de Orendain de la escena política señala el acceso de una nueva generación de ministros, aunque durante un tiempo permanezcan algunos de la antigua hornada, próxima ya su extinción. Tras el paso interino de José Patiño por el ministerio de Estado, el sucesor de Orendain fue Sebastián de la Cuadra y Llarena, perteneciente a uno de los linajes de Vizcaya que tomaton el apellido del barrio de la Cuadra, ayuntamiento de Musques, en el valle de Somorrostro. Parece que había empezado también como paje de Grimaldo, y que fue, a continuación, protegido del mismo Orendain. El hecho es que había ido ascendiendo en el ministerio de Estado hasta la primera mesa, para acceder desde ahí a una de las Secretarías de la Cámara de Castilla antes de llegar al ministerio -o Secreteraría del Despacho- de Estado.

En medio de esta fuerte tendencia a la profesionalización de la vida política, destacan figuras como la de Baltasar Patiño o el marqués de Torrenueva; porque, sin ascender por las mesas de ningún tipo de Secretarías como otros ministros, ambos habían desempeñado cargos relacionados con sus respectivos ministerios antes de acceder a ellos. Baltasar Patiño era señor y marqués de Castelar, de familia gallega y ligada a la administración de los Austrias en Milán. Fue Intendente del ejército durante la Guerra de Sucesión, después en Aragón y, desde ahí, alcanzó el ministerio de la Guerra en 1721, con Grimaldo. Mateo Pablo Diaz, marqués de Torrenueva, accedió más tarde al ministerio de Hacienda, en 1736, algunos meses antes de que llegara al de Estado Sebastián de la Cua$\mathrm{dta}$, con quien va a continuar hasta 1739. Desde 1715 venía desempeñando una serie de cargos hacendísticos importantes, entre ellos la dirección de las Aduanas y de la Renta General de Salinas en el reino de Sevilla, donde pertenecía a una de las familias principales de la capital ${ }^{12}$.

Similares a éstos últimos son los casos de José Patiño, Campillo y Ensenada, que van llegando a Guerra, Marina e Indias y Hacienda en la etapa de Sebastián de la Cuadra en Estado, a partir de 1736. José Patiño, hermano menor del mencionado marqués de Castelar, había estudiado en el Seminario jesuíta de Milán y en el de Roma. Pero, decidido a abandonar la cartera eclesiástica, había colaborado durante los últimos años de la Guerra de Sucesión como Superintendente General de Extremadura y de Cataluña. Como tal, había mostrado sus cualidades en los campos de la administración, la hacienda y la política, especialmente cuando llegó la hora de suprimir los fuetos catalanes e imponer allí un nuevo sistema contributivo, el del Catastro. Después había sido Intendente

12 Garcia CArraffa, op. cit., t. 27, y AHN, Est, Leg. 3496.

Hispania, LIX/1, núm 201 (1999) 193-215 
General de Marina; desde esta plaza su colaboración había seguido resultando indispensable en la organización de diversas escuadras destinadas a conseguir territorios italianos para los hijos de Isabel de Farnesio, Carlos y Felipe. De ahí, pues, su ministerio múltiple, con Hacienda, Guerra y Marina e Indias, más dos años en Estado ${ }^{13}$.

La vida de José Campillo muestra, una vez más, la importancia que tenía la práctica de poner los hijos al servicio de alguien que pudiera educarlos y protejerlos. Había nacido en Aller, en el seno de una familia hidalga asturiana de escasos recursos. Pero, aún adolescente, había ingresado al servicio de un prebendado de Córdoba que le envió durante unos años a la Universidad de aquella ciudad. En 1715 era ya secretario del Intendente General de Andalucía, el cual le hizo ingresar en el cuerpo de contadores de la atmada. Tuvo que destacar entre los contadotes, porque Patiño estuvo recurriendo a él hasta que, en 1736, le pudo ascender a Intendente de la marina y del ejército. Antes de llegar a su triple ministerio fue aún Intendente de Aragón ${ }^{14}$.

Zenón de Somovedilla, nacido en 1702 y después marqués de la Ensenada, era de origen riojano y de ascendencia hidalga probada por su ingreso en la Orden de Calatrava. Sabemos que estudió primeras lettas y humanidades en Santo Domingo de la Calzada; pero, como afirma su biográfo Rodriguez Villa, faltan noticias verídicas sobre el período posterior de su vida. Uno de los «relatos» existentes sitúa a Somovedilla en una casa de comercio de Cádiz, donde habtía logtado colocarlo un sacerdote tío suyo. Lo cierto es que allí le conoció Patiño cuando, como Intendente Genemal de Marina, fue a esa ciudad para preparar una expedición contra el asedio de los moros a Ceuta. ¿̇ue una opetación de patronazgo cuyos vinculos desconocemos, o simple reconocimiento de una capacidad poco común? En cualquier caso, nombró al joven Somovedilla oficial supernumerario del ministerio de Marina. Esa plaza más que modesta marca, sin embargo, el inicio de de la carrera de Ensenada; porque, ascendiendo en la escala hasta llegar a primer oficial, ocupó a continuación puestos diversos entre los que destacan el de Comisario de Marina en Cádiz o en el Ferrol; los más importantes fueron otros ligados a la preparación y otganización de escuadras destinadas a la toma de Orán o, más definitivo, a la conquista de Nápoles y Sicilia para el púncipe don Carlos, y de Parma, Plasencia y Guatalla para el infante don Felipe. Por esa vía legó, al morir Campillo en 1743, a ocupar los tres ministerios de Hacienda, Guerra y Marina e Indias 15 .

13 Cfr. en A. Rodriguez VILLA: Patiño y Campilla. Reseña bistório-biográfica, Madrid, 1882, pp. 11 y ss.

14 Quien más información proporciona sobre la vida de Campillo es su secretario Jorge Argandi, que constituye a su vez otro ejemplo significativo de ascenso profesional al servicio de algún personaje: Cuando estudiaba en el Seminario de Nobles de Lioma(Toscana), Argandi fue requerido por Campillo, entonces Intendente General del ejército, para pasat a su servicio. El vínculo lo proporciona la amistad de dos familias: La de los parientes gaditanos de Argandi y la de Campillo, con ayuda económica, además, de la primera al segundo en su carrera profesional. (Ver. en Ibid, pp. 131 yss)

15 Cfr. en A. Rodríguez Vilia: Don Zenón de Somovedilla, margwés de la Ensenada, Madrid, 1878.)

Hiparaia, LIX/1, núm. 201 (1999) 193-215 
La organización de las nuevas Secretatías del Despacho se inició desde el momento de la creación de las dos primeras en 1705 . Así, un real decreto del 19 de enero de 1706 informa que, en la de Guerra y Hacienda a cargo de José Grimaldo, han sido ya nombrados ocho oficiales. Tanto ellos como los de la otra Secretaría -la de "todo lo demás» a cargo de P.C. Fernández del Camporeciben, como protección, la categoria de "criados» de Su Majestad y, con ello, una cierta estabilidad en el empleo. El primer rasgo a destacar consiste en la identidad del modelo organizativo - tan sencillo, por lo demás- con el de las antiguas Secretarías de los Consejos. Coinciden los orígenes sociales de los oficiales, las vías y los requisitos de acceso; coinciden el ascenso de los oficiales desde las últimas a las primeras mesas a lo largo de los años y la mejora también paulatina de sus retribuciones; y coincide la salida con el ascenso final a Secretario de una de las oficinas de los Consejos, eso sí, en competencia con los oficiales que también en ellas van llegando a los primeros puestos. Lo que no coincide es la dimensión de las nuevas Secretatías del Despacho con la que tenían antes las de los Consejos. En un principio, el número de oficiales gira, en los ministerios, en torno a ocho, o bien menos según la relevancia de cada una de esas Secretarias del Despacho; de todas formas, ese número irá después aumentando con la importancia posterior de cada uno de los ministerios, y también según la habilidad de uno u otro ministro para convencer al rey de la necesidad de ampliar su propio departamento. A finales de 1714, justo antes de ser reformada y reducida, la Secretaría del Consejo de Estado tenía once oficiales de plantilla y otros nueve supernumerarios, además de cuatro traductores ${ }^{16}$. Lo que sucede ahora es que los supernumerarios y entretenidos, si no desaparecen por completo en las Secretarías de Consejos y en los ministerios, se reducen considerablemente, y no figuran ya en las plantillas ni en los presupuestos de esas instituciones; si se mantienen, se debe a las ventajas de disfrutar de una asistencia gratuita y a las del aprendizaje práctico en la profesión administrativa.

Como es sabido, la situación creada tras la Guerra de Sucesión llevó a la desaparición de algunos Consejos (Aragón, Flandes, Italia) y, con éllos, a la de sus Secretarías. Ni los unos ni las otras desaparecieron siempre, sin embargo, de un plumazo. Siguiendo una tendencia de la época, el de Italia se mantuvo mortecino, prácticamente inactivo, hasta la extinción total de sus consejetos. Es lo que explica la conservación paralela de una muy pequeña Secretaría de Italia, destinada, al menos, a custodiar los papeles del antiguo Consejo. En 1717, las cuatto antiguas Secretarías que había llegado a tener el Consejo de Italia- las de Nápoles, Sicilia, Cerdeña y Milán - se redujeron a una sola con cuatro oficiales escogidos entre los mejores de las cuatro mencionadas. Al fallecer el último de esos oficiales en 1746, los papeles pasaron definitivamente al ministerio de Estado ${ }^{17}$.

16 AHN, Est, Leg. $3.439, n^{\circ} .5$.

17 AHN, Est, Leg. $3496, n^{\circ} .5,6,7$ y 8 .

Hippania, LIX/1, núm. 201 (1999) 193-215 
Las Secretarias de otros Consejos se mantuvieron con ellos; pero, repartida ahora la administración con los ministerios y en alza éstos frente a la decadencia de los antiguos organismos colegiados, en 1717 se recortó el número de oficiales de las antiguas Secretarías. Ninguna de ellas iba ya a sobrepasar el previsto para las de los ministerios. Así, las tres de la Cámara de Castilla (Patronato, Gracia y Aragón), quedaron con seis o siete cada una; las dos de Indias (Perú y Nueva España), con seis cada una; y la de Ordenes con ocho.

Ahora bien, la reforma y la reducción más espectaculares fueron las que afectaron precisamente a las más prestigiosas de entre las antiguas Secretarias: Las dos de Estado (Norte e Italia) y las dos de Guerra (Tierra y Mar). Ya la anterior reforma administrativa de 1691 -más aspiración o intento que teforma de hecho- había dejado once oficiales y nueve supernumeratios en la Sectetaría de Guerra de Tierra y un número algo menor en la Secretaría de Mar. Los ingresos de ambos Secretarios, especialmente los del de Tierra, eran de gran cuantía por sumarse a sus sueldos buena parte de lo aprehendido en el contrabando de comestibles y de los derechos de los despachos de partes ${ }^{18}$. Eran retribuciones sólo comparables a las de los dos Secretarios del Consejo de Estado, de más de 60.000 reales al año cada uno. No es de extrañar que cuando, preparando ya las reformas, en 1701 se pida información al Consejo de Estado sobre sus Secretarías, empleados y sueldos, la respuesta resulte casi agresiva: Fue un alegato sobre la importancia de los Consejos como modelo ante otras naciones, sobre su propio prestigio, y sobre la necesidad de que sus Secretarios y empleados estuvieran bien dotados y considerados ${ }^{19}$. Pero el proceso de reformas continuó su camino: En 1706, Felipe $\mathrm{V}$ fusionó las dos Secretarías de Guerra heredadas, la de Tierra y la de Mar; en 1714 quedaron también unificadas las dos Secretarías de Estado (Norte e Italia) en una fusión que, como ha señalado Escudero, es paralela al arrinconamiento definitivo del Consejo de Estado frente al peso del nuevo ministerio de Estado, mayor que el de los demás; y en 1717 las Secretarías de Estado y de Guerra acabarían fusionándose en una sola con un Secretario y seis oficiales, tres de ellos procedentes de Estado y otros tres de Guerra ${ }^{20}$.

Realizada la fusión, el nuevo Secretario de Estado y Guerra fue Juan de Elizondo y Echenique, de familia hidalga del valle del Baztán, caballero de Santiago y con una larga carrera administrativa a sus espaldas. Al recibir el nombramiento en 1717 llevaba ya 46 años de servicios: Primero, en las dos Secretarias de Mar y de Tierra; como Secretario en la de Guerra reunida después, desde 1706; y con el mismo alto cargo en la también reunida de Estado desde 1714. Su retribución en la nueva de Estado y Guerra fue de 74.000 reales al año, comparable a la de los primeros ministros de Felipe V ${ }^{21}$. Elizondo llegó a ser todo un

18 AHN, Est, Leg. 2835, reproducido por J.A. Escudero en Los Secretarios de Estado y del Despacho, op. cit, IV, pp. 1029-1032.

19 AHN, Est, Leg. 3497.

20 Ibid., y 3496.

21 AHN, Santiago, exp. n ${ }^{\circ}$ 2608; Est, Leg. 3439, $n^{\circ} .5$, y su título de Secretario en 1714, reproducido por Escudero en Los Secretarios.., op. cit. III, pp. 669 y ss. 
personaje: Tuvo a su único hijo de paje de Felipe $\mathrm{V}$ durante diez años; a continuación, logró que se le admitiera como oficial en el regimiento de caballería del Príncipe, pero a sus propias expensas, con el gasto que ello suponía cubierto por el sueldo del padre. Parece claro que el prestigio aportado a la familia compensaba el coste con creces. En cualquier caso, Elizondo pidió también, por intercesión de Grimaldo, empleo retribuido para su yerno, un criollo peruano 22 .

La Secretaría de los Consejos de Estado y Guerra siguió siendo un puesto codiciado en la carrera administrativa. En 1738, tras la muerte de Elizondo, le va a suceder Casimiro de Uqtáriz comendador de Usagre en la orden de Santiago. Casimiro, primer marqués de Uztáriz, era hijo de Gerónimo, autor de la Theorica y Práctica de Comercio y de Marina(1724). Tanto el padre como después el hijo habian ido ascendiendo a lo largo del escalafón del nuevo ministerio de la Guerta. El padre era persona respetada, a quien se pedían informes secretos cuando ocupaba la primera mesa. En 1730, Casimiro era también Secretario de la Junta de Comercio y Moneda; es cuando su padre le había recomendado a Orendain para que se le asignara una buena Secretaría de Consejos al terminar su larga etapa en el Despacho de Guerta. La petición insistía en las cualidades del hijo, pero lo hacía ante todo en el carácter de favorecedor de la familia que tenía el entonces ministro de Estado: «Para que tengamos este nuevo y especial motivo que aumente las obligaciones que profesamos a V.E.» Casimiro de Uztátiz consiguió entonces la Secretaría de la Sala de Millones, una de las del Consejo de Hacienda y también uno de los escalones iniciales en la peregrinación por las Secretarías de los Consejos. Su próximo ascenso será ya la de Estado y Guerra en $1738^{23}$.

Pero, desaparecido Elizondo, algo va a cambiar entonces en esa Secretaría. El ministro de la Guerra y presidente del Consejo del mismo nombre, duque de Montemar, se queja del atraso que hace tiempo padecen los asuntos militares en la Secretaría conjunta y, sin menci nar el nombre de Elizondo, hace sin embargo una crítica de su gestión por desfasada. A continuación consigue que Uztáriz tenga asistencia y voz en el Consejo de Guerra, y que siga al Rey en sus desplazamientos, como había sucedido antes de la reforma de 1717. Refuerza, pues, la dedicación de la Secretaría de Estado y Guerra hacia estos últimos asuntos y hacia el Consejo de Guerra frente al de Estado. Es una prueba más de la decadencia de éste último, absorbidas como habían quedado sus posibles actividades por el ministerio del mismo tamo. Montemar propone, además, como le es también aceptado, que los ingresos del Secretario, excesivos como reminiscencia de otras épocas, se reduzcan a la cantidad, tampoco despreciable, de 60.000 reales al año. Siguió siendo, a pesar de todo, uno de los puestos más altos en la carrera administrativa. Al morir Casimiro de Uztáriz en 1751, recaerá en Agustín Pablo de Ordeñana, gracias, sobre todo, a su fidelidad y amistad política

\footnotetext{
22 AHN, Est, $\operatorname{Leg} 3496, \mathrm{n}^{\circ} .48$ y 58.

23 AHN, Est, Leg. 3496, $n^{\circ}$. 11, y el titulo de C. de Uztáriz, reproducido por Escudero en op. cit., III, pp. 675-676.
}

Hispania, 1IX/1, núm. 201 (1999) 193-215 
con el entonces poderoso marqués de la Ensenada. Ahora bien, en el escalafón de esa Secretaría iba ascendiendo, desde 1745 , otro miembro de la familia Uztátiz: Lorenzo Fajardo y Uztáriz, sobrino de Casimito y nieto de Gerónimo, en quien coincidian los servicios y méritos contraídos durante varias generaciones por sus mayores maternos y paternos ${ }^{24}$.

Era inevitable que, al menos al principio, las reformas de Felipe $V$ provocaran conflictos entre las instituciones antiguas y las nuevas, entre los Secretarios de los Consejos y los de Estado y del Despacho, especialmente entre los primeros y el ministro de Estado. Hay que tener en cuenta que todas las propuestas, tanto de los Secretarios de los Consejos como de los ministros, sobre entrada de nuevos oficiales y ascensos en sus oficinas respectivas quedaron pronto mediatizadas por el titular de Estado. Es él quien traslada esas propuestas al rey, él quien le informa y le aconseja. Así, cuando uno de los Secretarios del Consejo de Indias trata de aprovechar una vacante para aumentar hasta trece y un archivero el número de sus oficiales, la respuesta es tajante: Las Indias- se le dice- ya no se gobiernan sólo por ese Consejo; porque todo lo referente a guerra, armadas, flotas, galeones y hacienda se lleva en el ministerio de Marina e Indias, y la Secretaría del Consejo tiene más que suficiente con ocho oficiales ${ }^{25}$.

En cualquier caso, la reducción de oficiales en las Secretarías de los Consejos dejó, desde 1717, a bastantes de ellos sin empleo. Algunos, los mejores o los mejor situados, pasaron a los nuevos ministerios; alli reiniciaron sus carreras, algunos desde muy abajo. Es el caso de Francisco Campo de Arve que, tras muchos años en la antigua administración y habiendo llegado bastante arriba, vuelve a empezar en la última mesa del ministerio de Justicia en 1717.26 Pero también los hubo que quedaron en espera de ser teabsorbidos en el futuro, al hilo de vacantes en uno u otro tipo de Secretarias. Felipe V ordenó que se les pagara mientras tanto la mitad del sueldo, aunque las quejas de los interesados indican que no siempre fue así. Desde la Secretaría de Estado y Guerra, Juan de Elizondo reivindicó su derecho a no admitir forzosamente en las vacantes a estos "creformados" cuando le constaba su escasa capacidad. Y, haciendo gala de su latga experiencia en la administración y de lo que había visto en ella, defendió el criterio de la aptitud como corrector del de la estricta antiguedad. A Elizondo le fue permitido aplicar sus ideas, tal era el prestigio que arrastraba del reinado anterior, el de Carlos II. Pero, nada más llegar Casimiro de Uztáriz, en 1738, reinsertó a dos antiguos oficiales sin empleo desde $1717^{27}$.

24 AHN, Est, Leg. $3496, n^{\circ} .11,62,67$ y $\sin n^{\circ}$.

25 AHN, Est, Leg. 3497.

26 AHN, Est, Leg. 3497.

27 AHN, Est, Leg. 3496, nº 57 y 60; y ejs. de desplazados en Ibid, Leg. 3497.

Una situación heredada del siglo anterior y que llevatía algún tiempo normalizar fue la de las porterías de los Consejos, al menos las de algunos de ellos. Como forma de retribuir determinados servicios a la monarquía, se habían ido concediendo en su día en los Consejos de Estado, Guerra e Italia, por ejemplo. Pero esas porterías habian sido cedidas "por dos vidas", 
$\mathrm{El}$ ascenso de los oficiales mayores de uno u otro tipo de Secretarías a Secretatios de los Consejos fue otro motivo de competencias, aunque no por mucho tiempo; porque pronto quedaron relegados los de los Consejos a favor de los de los ministerios: Preferir a los primeros -se afirma en un informe sin firma-sería un perjudicialísimo ejemplan» y «del mayor desdoro" para las nuevas Secretarías del Despacho; pues se daría «a entender no haber en ellas sujetos dignos... por su mérito, ...por su antigüedad ni por sus circunstancias» ${ }^{28}$. De ahí que no tardara en ser ésta la política normal de ascensos; y que, para los oficiales de los Consejos, queden otras Secretarías de segunda fila, como la de la Junta de Dependencias de Extranjeros, por ejemplo ${ }^{21}$. Un buen final de carrera administrativa, cuando se quiere premiar los servicios prestados por determinados individuos, consiste en otorgarles una plaza de capa y espada en alguno de los Consejos que no sea el de Castilla.

Los conflictos iniciales entre autoridades e instituciones fueron, a veces, graves. Los tuvieron el marqués de Torrenueva e Iturralde como ministros de Hacienda, por breve que fuera el paso de ambos pot ese ministerio. Torrenueva encontró grandes resistencias al pedir, en 1737, la presidencia del Consejo de Hacienda para sí mismo y los ministros que le sucedieran. Ese Consejo llevaba bastante tiempo sin gobernador, mientras la solución de los asuntos, contenciosos o no, se retrasaba allí sine die. La dejadez era tal -según Torrenueva- que no conseguía renovar los contratos más urgentes de la Real Hacienda, los de arrendamiento de los impuestos; los hombres de negocios se negaban, pues no veían garantizados sus derechos por el Consejo. Al tiempo que insistía en la gravedad de la situación, el ministro explicaba la conveniencia de hacer coincidir ambos cargos en la misma persona por simples razones de eficacia: Es preciso -decía- que "quien trate el punto de medios con los recaudadores», pueda "hacerles comprenc'er que quien los pide es capaz por sí de cumplir lo que ofrece, respecto di: las facultades que Su Majestad le confiere». Torrenueva elevó todas sus propuestas a través del ministro de Estado Sebastián de la Cuadra, ya marqués de Villarias por entonces. Pero las resistencias debieron de ser fuertes. Ni siquiera se le aceptó la persona que propuso como Gobernador de Hacienda al serle negado el cargo a él mismo. Torrenueva quería alguien versado en la práctica de Hacienda, no un "togado, pues sólo son buenos para votar pleitos de Justicia»; los conseje-

prorrogables a veces a más generaciones, y con la posibilidad de nombrar sustitutos que las atendieran. En Estado había dos, y otras dos en Guerra. La retribución anual era de 600 ducados, 300 de los cuales habian sido de plata doble en el siglo XVII. Conseguir una de ellas era una forma de contribuir al porvenir de un hijo, a veces de disponer de una dote para una hija, dada la posibilidad del teniente o sustituto de los cargos enajenados. No sólo se respetaron las existentes en tiempos de Felipe V,sino que se concedieron las que seguian pidiéndose. No se acabó con tales cargos hasta 1754-1758. ( $A H N$, Est, Leg. 3496, $\mathrm{n}^{\circ} .16,32,35 \mathrm{y} \sin \mathrm{n}^{\circ}$ ).

28 AHN, Est, Leg. $3496, n^{\circ} .89$.

29 AHN, Est, Leg 3496, n'. 60 .

Hihpontu, LIX/1, núm. 201 (1999) 193-215 
ros querían alguien de «reconocida dignidad ${ }^{30}$. El ministerio y el gobierno del Consejo de Hacienda coincidieron, finalmente, en la misma persona desde 1739 , con Juan Bautista de Iturralde. Salvo el de Castilla, que se mantiene en un plano superior, los demás Consejos tenían que ser y fueron antes o después presididos por los correspondientes ministros.

Iturralde tuvo que arrostrar, por su parte,, las iras del Cardenal Gobernador del Consejo de Castilla. Junto a otras gentes poderosas a quienes perjudicaban las medidas tributarias del nuevo ministro de Hacienda, había creado un pequeño grupo de oposición, fuente de habladurías y descalificaciones. La reprimenda real, lanzada al Gobernador a través de su ministro de Estado, fué rápida y eficaz: Se le recordó que la «suma autoridad» que ejercía al frente del Consejo de Castilla «no le había sido concedida para desdorar a (los) ministros...que, con tan distinguida inmediación, sirve(n) a...los reales piés» ${ }^{31}$. Era el triunfo de los ministros frente al que, tradicionalmente, había encarnado la figura de mayor prestigio después del Rey, el gobernador o presidente de Castilla. No en vano, $\mathrm{y}$ aunque no sea más que un signo puramente extetno, las oficinas ministeriales se fueron acomodando desde el principio en dependencias del palacio real, junto al monatca con quien despachaban personalmente los ministros. A los Consejos se les ordenó, en cambio, reunirse fuera, en el palacio propiedad del duque de Uceda, en la calle Mayor.

III

Un problema que se va a plantear pronto en los ministerios será el de la estabilidad de sus oficiales ante los cambios ministeriales, frecuentes con Felipe V. La facultad de sustituirlos por otros estuvo permitida a los ministros hasta 1721 , mientras aquellos oficiales pudieron también tener más de un empleo. Terminada esta posibilidad en 1717, con ella acabó igualmente, en enero de 1721 , la libertad de los ministros para cambiar a su gusto los oficiales ${ }^{32}$. Un real decreto de esa fecha, repetido en mayo de ese año, en 1724 y en 1726 , otorga estabilidad y derecho a un ascenso regular a esos empleados: No es justo-afirma el decreto, aunque tampoco parece que pudiera resultar prudente-que, después de haber estado manejando negocios de tanta consideración, se vean obligados a mendigar; desde entonces sólo podrían ser expulsados por «el motivo de insuficiencia, demérito o delito" y, claro está, con aprobación real. El nombramiento como secretarios del monarca, firmado por el mismo, constituiría desde entonces un título de garantía; la mayor parte de ellos etan, además,

30 AHN, Est, Leg. 3496, n. 91 y 92. Al menos Juan de Dios Río González, marqués de Campoflotido, había presidido el Consejo de Hacienda mientras, durante algun tiempo, fue ministro de ese ramo con Grimaldo antes de la abdicación de Felipe V en su hijo.

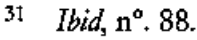

32 Con todo, siguió liabiendo empleados con más de una plaza; no fueron, sin embargo, más que excepciones confirmando la regla, especialmente si dejaban de proporcionat algún ahorro a la Hacienda porque lograban percibir dos sueldos.

Hispaniz, LIX/1, nứr. 201 (t999) 193-215 
oficiales con ejercicio de decretos, con facultad para refrendar los del Rey; sólo las últimas mesas estaban ocupadas por los oficiales de registro y de partes, especialmente dedicados a las reclamaciones que iban llegando de fuera, $y$ a veces por algún escribiente.

Lo que sí quedó a la libre decisión de los ministros fue la selección de los nuevos oficiales llamados a cubrir las plazas libres -siempre las últimas- por muerte y ascenso de los demás; había de preceder, sin embargo, la aprobación real, normalmente decidida con el ministro de Estado. Ministros hubo que trataron de no respetar la norma; pero, como era de esperar, el control quedó asegurado a través de la protesta de los oficiales perjudicados. Es lo que sucedió cuando, en 1724, Fernando Verdes Montenegro, ministro de Hacienda, se empeñó en meter en la primera mesa a un hermano suyo, despreciando con ello el derecho al ascenso de los oficiales existentes desde hacía años. El hermano tenía experiencia, pues había desempeñado algunos empleos para la Real Hacienda en el Reino de Valencia; pero tuvo que contentarse, finalmente, con la última mesa del ministerio. El poder de los ministros para ejercer el patronazgo quedaba, por lo tanto, bastante limitado ${ }^{33}$.

Baltasar Patiño, marqués de Castelar, intentó también extralimitarse al llegar al ministerio de la Guerra en 1721, y tuvo igualmente que ceder. La supresión de las Secretarías del Consejo de Guerra, cuando sólo la de Tierra había llegado a tener 15 o 16 oficiales, recargaba en exceso el trabajo en ese ministerio. De ahí que tuviera mayor número de empleados que otros, con 6 oficiales de decretos, 2 de registros, un escribiente y un empleado contratado por un año. En aquellos momentos había, además, una vacante en las mesas de decretos. La aspiración de Castelar consistía en aumentar su departamento con dos oficiales más, pero también en sustituir a dos de los existentes por otros dos elegidos por él. De forma que proponía, en conjunto, a cinco indivíduos de su propia conveniencia. Es lo que evitó la intervención de Grimaldo, ministro de Estado: Se le aceptó el aumento de dos mesas, las últimas, para otros tantos escribientes que él pudo elegir; pero no se le permitió expulsar a nadie y, en la vacante, se le obligó a encajar al contratado que, en opinión de Grimaldo, había resultado ser el más capaz en aquella Secretaría del Despacho ${ }^{34}$.

Entre los beneficiarios del patronazgo ministerial, por limitado que fuera, siguen contándose los pajes, especialmente los de los propios ministros. Con frecuencia, colaboran en lo posible en la Secretaría del Despacho correspondiente y, por cómo se expresa uno de los pajes de Patiño, consideran que su «ascenso regulan» es el de la última mesa de esa Secretaría. En la de Estado al menos, se les da pequeñas gratificaciones en fechas señaladas, como las de $\mathrm{Na}$ vidad. Los Secretarios de los Consejos son, en cambio, los que parecen seguir haciendo asistir a alguno de sus hijos a las tareas diarias de su propia Secretaría,

33 AHN, Est, Leg 3497.

34 Ibid. De todas formas, siempre hay alguna excepción que confirma la regla en este tema del ascenso riguroso y de la entrada por abajo. También, aunque no sea frecuente, hay casos de oficiales que pasan de un ministerio a otro.

Hippanio, LIX/1, nứr. 201 (1999) 193-215 
activo importante después para cualquier empleo administrativo. Pero también lo sigue siendo la experiencia adquirida en otras esferas administrativas, desde la militar a la eclesística, la de los virreinatos americanos, o incluso la privada ${ }^{35}$.

El mismo predominio de la experiencia es el que rige en el inicio de una carrera diplomática cuando no se pertenece a una familia de la nobleza titulada. Porque son generalmente los titulados quienes ocupan las embajadas, empleando a veces parte de sus recursos propios en mejorar la representación española en el exttanjero. Los simples hidalgos, a veces los mismos titulados, procuran encontrar las influencias adecuadas para colocar a sus hijos como kjóvenes de lenguas): Estos habían de ser -como repetirá más tarde una orden de Carlos III- «jóvenes instruidos y de distincióny; es decir, cultivados en la lectura y, a ser posible, en el arte, pero, sobre todo, acostumbrados al estudio de los idiomas. Son enviados a las diferentes embajadas españolas; residen en ellas con todos los gastos pagados, pues no perciben sueldo alguno o sólo cantidades mínimas. Sus obligaciones consisten en irse habituando a la lengua y las costumbres del país correspondiente, así como al trabajo de la Secretaria de la embajada, donde hay siempre algunos -pocos- oficiales. Es el inicio de una carrera diplomática que, a través de ascensos por esas oficialías, puede llevarles al puesto de Secretario de embajada; y también se va ascendiendo, desde las Secretarías menos importantes, hasta las de las primeras embajadas, las de Londres, París, Viena y Roma ${ }^{36}$.

El primero y principal de los ministerios era el de Estado, destacando sus oficiales sobre los demás junto al ministro de ese ramo. Los sueldos eran, sin embargo, iguales o muy similares de uno a otro ministerio. Se movían entre los 35.000 reales/año que solían percibir los oficiales mayores -el más antiguo y el menos antiguo cuando había dos- y los 15.000 del séptimo y el octavo. La diferencia se amplió cuando, en 1742 , Felipe V concedió a los oficiales mayores de sus ministerios los complementos más habituales en la época, los «gajes y casa de aposentos, que suponían una mejora en la tetribución. En la medida en que fueron institucionalizándose en los ministerios, los archiveros y sus oficiales, por no hablar de los simples escribientes, ganaron siempre menos; un archivero podía a veces alcanzar los 11 ó 13.000 reales por año, pero la tetribución de los escribientes oscilaba entre los 1.000 y los 8.000 reales. Hasta la llegada de Carlos III no habría una subida generalizada, subida que se extendió, por lo menos, a los magistrados de los Consejos.

En cualquier caso, y por los datos hallados para algunas Secretarías de los Consejos, los sueldos de sus oficiales parecen haber sido más bajos que los de sus colegas ministeriales. Ello a pesar de que las reformas impuestas por Felipe $\mathrm{V}$ a estas Secretarías mejoraron, en términos generales, las retribuciones anteriores a cambio de la reducción de empleados; y a pesar de que los sueldos de

35 AHN, Est, Legs 3497 y $3496, n^{\circ} 59$.

36 AHN, Est, Legs. 3427, 3430, 3436, 3449 ó 3450, p.e. Los consulados han sido estudiados por Jesús PRADEII NADAL: Diplomaciay comercio. La expansion consular española en el siglo XVIII, Universidad de Alicante, 1992.

Hispania, LIX/1, núm 201 (1999) 193-215 
sus jefes, los Secretarios de los Consejos, siguieron moviéndose en otros niveles. Así, tras la reestructuración de 1717, el primer oficial de la Secretaría de Ordenes percibía tan sólo 18.000 reales, el de Gracia de la Cámara de Castilla 16.000 , y los de las últimas mesas en una u otra 4 ó 2.000 reales al año. Los sueldos en la Secretaría de Estado y Guerra eran claramente mejores, pero también menores que en los ministerios y a pesar de los mencionados 74.000 reales de su Secretario Elizondo ${ }^{37}$.

Las retribuciones que reciben los ministros son ya de otro orden; son bastante superiores incluso a la del gobernador del Consejo de Castilla. El prestigio de este personaje bajo la monarquía austriaca había sido suficiente como para considerarle la segunda figura del reino, con sólo el monarca por encima aunque en la práctica quedara después supeditado a los validos. Tampoco su poder ni su influencia podrán compararse con los de los ministros de los Botbones, y lo mismo acabará sucediendo con su retribución. Al principio, cuando surge la figura de los ministros con Felipe $\mathrm{V}$, su retribución queda fijada en 120.000 reales, aunque de momento no conozcamos qué complementos se le sumaban entonces. Pero sí sabemos que, no mucho tiempo después, Sebastián de la Cuadra, marqués de Villarias y ministro de Estado entre 1736 y 1746, percibía un total de 335.640 reales: A los 120.000 del sueldo se sumaban otros 118.000 reales para su mesa, además de diversas cantidades menores. Los complementos a los sueldos de los ministros variaron a lo largo del siglo XVIII; mas no dejaron por ello de ser considerables, especialmente los correspondientes a los ministros de Estado ${ }^{38}$.

$\mathrm{Al}$ ministerio de Estado le correspondió desde el principio la política exterior, que en el siglo XVIII conservaba aún un papel primordial. Bajo la dirección del ministro, el cometido más importante de sus oficiales consistió siempre en da correspondencia con las naciones amigas y los asuntos relativos a las enemigas». Pero, también desde el principio, hubo ottos campos al cargo de ese ministerio. Entre ellos destaca la Superintendencia General de Correos, Postas y Caminos, que dependía del ministro de Estado desde que, a principios de siglo, pasó de manos privadas a las del Estado; era, sin embargo, un servicio

${ }^{37}$ AHN, Est, Leg. 3439, n $n^{\circ} .5,21,44,63 ;$ Leg. 3496, no. 58 y Leg. 3497. Las plantillas y sueldos del ministerio de Justicia han sido cuidadosamente reconstruidos por R. GómEZ-RrvERO: Las orígenes del ministerio de Justicia, op. cit.- Con Catlos III no llegó sólo la mencionada subida generalizada de los sueldos. Llegaron también, en 1763, los Montepíos de viudedad y horfandad; el primero fue el militar, creado por Esquilache, extendièndose a continuación a los Consejos, tribunales y organismos de la administración. La práctica dominante hasta entonces consistia en conceder, de una sola vez, alguna cantidad a las viudas o huérfanos más necesitados que acudian al Rey.

38 AHN, Est. 3439, n. 34; tambien, M. Victoria LóPEz-CORDÓN: La primera Secretaría de Estado: la Institución; los hombres, y su entorno (1714-1833), en Revista de la Universidad Complutense, $\mathrm{n}^{\circ} .116,1979$, pp. 16-39; de la misma: Oficiales y caballeros. La carrera administrativa en la España del siglo XVIII, en El mutudo bippánico en el siglo de has tuces, Madrid, 1996, T. II. Cfr. también, M.T. NAvA RoDRiGUEz: La Secretaria de Hacienda en el Setecientos español, en op. ait; y G.A. Franco RuBIO: Reforma administrativa y nuevas instituciones: Las Secretarias de Guerra y Marina del siglo XVIII, en op. cit., t. I.

Hipania, LIX/1, núm. 201 (1999) 193-215 
con sus propias oficinas, sus propios oficiales y hasta su propio juzgado. Asuntos al cargo de los oficiales del ministerio fueron, en cambio, los relativos a los Reales Sitios y a las jornadas del rey fuera de la capital, a los infantes y su economía, a las concesiones de grandezas y a la supervisión de la Orden del Toysón o de la Junta de Dependencias de Extranjeros, a las acequias reales de Aragón y del Jatama y a ottos campos que fueron cayendo bajo la competencia del ministerio a lo largo del siglo. El reparto de tantos temas entre las mesas y los oficiales de Estado no era fijo; iba variando, por el contratio, con los distintos ministros, con las relaciones diplomáticas hacia los distintos países, posiblemente también con las aptitudes de unos $\mathbf{u}$ otros oficiales en cada momento. De igual forma, en los diversos stepartimientos» que nos han quedado puede aparecer, o no, un oficial encargado de distribuir los papeles entre las mesas; o bien quedar, o no, las últimas explícitamente encargadas de resumir memoriales $\mathrm{e}$ instancias de parte, cartas de oficio y de particulares, de hacer todos los registros, copias y traducciones necesarias. A finales de 1790 , próximo ya el fin de su vida política, Floridablanca confesaba su preocupación por no haber dado aún con el reglamento y distribución del trabajo más adecuados para aquel ministerio ${ }^{39}$.

En las plantillas del de Estado, más que en las de otros ministerios, es frecuente la sucesión, incluso a veces la coexistencia, de miembros de una misma familia. Dada la forma de acceso, no es de extrañar esta formación de «familias administrativas». Un caso llamativo es el de los Mollinedo, dada su capacidad para extender su influencia a dos etapas distintas, con gobernantes y círculos de poder diferentes. Nicolás de Mollinedo y de la Cuadra entró como oficial en 1736, coincidiendo con la sustitución de Orendain por Sebastián de la Cuadra en ese ministerio. La madre y los abuelos maternos de Nicolás pertenecían a la misma familia y al mismo concejo vizcaíno que el nuevo ministro, al concejo de Musques. Poco después, en 1739 accedió también un hijo de Nicolás, Manuel, que sería promovido en 1746 a ministro de Marina en Cantabria con el grado de Comisario Ordenador. Pero es que mientras tanto, en 1738, había entrado Enrique de Mollinedo y Villanueva, natural del valle de Arcentales, en las Encartaciones de Vizcaya; pertenecía a otra rama Mollinedo, pero de familia vizcaína por ambos costados. Al entrar en el ministerio de Estado, contaba con ocho años de práctica en el uoficio de papeles» junto al mismo ministro ${ }^{40}$.

El primer Mollinedo, Nicolás, fue después, ya durante los últimos años de Fernando VI -tras la muerte de Carvajal y una vez derrocado Ensenada en 1754 protegido y colaborador del ministro de Estado Ricardo Wall. Llegado Nicolás a la oficialía mayor del ministerio y ascendido en 1758 a Secretatio del Consejo de Hacienda en su sala de Millones, Wall le reclamó de nuevo junto a él. Quetía el ministro, y lo consiguió del Rey, que, sin perder Nicolás la Secre-

$39 \mathrm{AHN}, \mathrm{Est}, 3439, \mathrm{n}^{\circ} 34$.

40 AHN, Est, Legs. $3439, n^{\circ} .34,40$ y $\sin n^{\circ}$.; $y$ Leg. 3449,1 . Sin proceder de Musques, hay casos como el de los Otamendi, de Guipúzcoa, cuya presencia en el ministerio de Estado sigue renovándose durante el reinado de Carlos III. (AHN, Est, Leg. 3433,1).

Hispania, LIX/1, núm. 201 (1999) 193-215 
taría, siguiera trabajando a sus órdenes directas y le acompañara en todos sus desplazamientos junto al monarca a los Reales Sitios. Tan complicada duplicación de cargos se habia producido ya alguna pero rara vez; requería, por supuesto, la compenetración personal y política del ministro con su oficial mayor, a quien deseaba conservar sin privarle por ello del ascenso correspondiente. No parece aventurado suponer que este Mollinedo se había identificado con la nueva política inaugurada por Wall; es decir, con la política ilustrada acentuadamente regalista, antijesuita y anticolegial, característica del largo reinado de Carlos III. En 1741 había obtenido ya Nicolás un hábito de Santiago. Hasta 1761-1762 no le llegaría el premio más preciado a ésta como a otras carreras al servicio de la monarquía, algún título de nobleza: Obtuvo entonces los de vizconde de Peñaflor y marqués de los Llanos, que agradeció lógicamente a Wall: Se muy bien - decía al ministro- que unada pued(o) merecer sin el favor y protección de Vuestra Excelencia,s ${ }^{41}$.

Cabría también aludir, por otro lado, a José Agustín del Llano y de la Cuadra. Procedente del ya tan mencionado concejo de Musques, entró en el mismo ministerio de Estado en 1741, para continuar después, ya con Carlos III, una brillante carrera en la que le seguiría su hermano Sebastián. Se puede mencionar igualmente a Nicolás de la Cuadra, que accedió al ministerio algo después que su primo Sebastián, y que había formado parte de la reducida Secretaría de Orendain, en 1724, junto a Felipe V durante el breve reinado de su hijo Luis I. Es preciso recordar algo ya dicho: Que Sebastián de la Cuadra habia empezado de paje de Grimaldo, pero que estuvo muy ligado después al siguiente ministro de Estado, Orendain, como éste lo había estado, por lo demás, a Grimaldo. El caso de de la Cuadra con Orendain, al ser ascendido el primero, en 1730, a la Secretaría de la Cámara de Aragón, constituye un precedente del de Nicolás de Mollinedo con el ministro Wall en 1758. Porque, por expresa petición del ministro al Rey, Sebastián de la Cuadra siguió desempeñando su labor en la primera mesa de Estado, junto a Orendain ${ }^{42}$. Es decir, que hay un grupo de poder a cuyo frente se van sucediendo Grimaldo, Orendain y Sebastian de la Cuadra entre 1714 y 1746 . A lo que habria que añadir el ministerio de Guerra y Hacienda, el más importante en su día, entre 1705 y 1714. Es lo que se ha llamado «el grupo de los vizcaínos», que dominaron a lo largo de cerca de 31 años, hasta la subida al trono de Fernando VI con Bárbara de Braganza.

No todas las "familias administrativas» de esas casi tres décadas son de origen vizcaíno. Hay casos que prolongan todavia a las antiguas de los Austrias. Lo demuestra, por ejemplo, el caso de Diego de Morales y su hijo Francisco Javier. La carrera administrativa del padre había empezado en 1690, en la Secretaría de uno de los Consejos de los Austrias; había sido a continuación uno de los oficiales elegidos por Grimaldo para su primer ministerio de Guerra y Ha-

41 Ibid, Est, Legs. 3439, nº. 28 y 3427,2, nº 20; Cons, Lib. 627 y Leg. 9984, nº 6; Pruebas de Santiago, exps. 5394 y 5396.

42 Pruebas de Santiago, exp. 4.729; Est., Legs. 3445, $\mathrm{n}^{\circ} 12,3497$ y $3496, \mathrm{n}^{\circ} .13$.

Hipania, LIX/1, núm. 201 (1999) 193-215 
cienda, donde Diego de Morales ocupaba el tercer puesto en 1706; después, al subdividirse los dos ministerios iniciales en otros cinco, había pasado al de Guerra, había ascendido allí hasta la primera mesa y había sido, más adelante, Secretario del Consejo de Ordenes, del de Indias y de la Cámara en lo referente a la corona de Aragón. Era la plaza que ocupaba al morir, en 1730. Entre los pretendientes a la misma se hallaba Francisco Javier de Morales que, sin méritos ni antiguedad suficientes por entonces, pretendia complementarlos, sin embargo, con los 40 años de servicio del padre, incluso con los méritos de su madre como camarera de la reina Mariana de Austria. En cualquier caso, él mismo habia ingresado en la Secretaría del Consejo de Indias en 1713, había seguido en la de Ordenes y se había incorporado luego, ya definitivamente, al ministerio de Estado. Era, además, caballero de Calatrava desde los ocho años, edad de ingreso en las Ordenes Militates que no constituía una excepción. La Secretaría vacante, la de Aragón, correspondió aquel año de 1730 a Sebastián de la Cuadra, con 25 años de antiguedad en el ministerio de Estado, siete de los cuales en la primera mesa del mismo. Francisco Javier de Morales inició algo más tarde su paso por las Secretarías de los Consejos; en 1740 había alcanzado ya de Gracia y Justicia de la Cámata de Castilla ${ }^{43}$. Iñigo de Torres y Oliveiro constituye un ejemplo más: Llegó en 1740 a la Secretaría de Patronato de la Cámara de Castilla, tras 31 años de servicio. Procedía del ministerio de Justicia, donde había ido subiendo hasta la primera mesa; después había seguido ascendiendo por las Secretarías de los Consejos. Al lograr la de Patronato en la Cámara, obtuvo, además, la última plaza para el mayor de sus cuatro hijos, Dámaso; junto a los méritos del padre, el mayor activo del jóven consistia en estar ya entrenado en el oficio gracias a su asistencia a las Secretarías ocupadas hasta entonces por su padre ${ }^{44}$.

No todo fueron, sin embatgo, familias y familiares en éste ni en otros ministerios durante la primera mitad del siglo. No hay más que repasar las plantillas de empleados que nos han llegado. En el de Estado aparecen, junto a otros nombres más o menos localizables, algunos de intelectuales conocidos. Es el caso de Agustín de Montiano, o el de Juan de Iriarte. Después, desde la llegada de Wall en 1754, van entrando personas de espíritu ilustrado; pero hay también una politización, la que se inicia con el derrocamiento de Ensenada y con el viraje anticolegial y antijesuíta que caracterizará al reinado de Carlos III. A pesar del orígen también vizcaíno de la familia materna de Montiano, su acceso en 1735, aún con Orendain, como el de Iriarte en 1742 con de la Cuadra, ejemplifican la temprana apertura del patronazgo oficial a intelectuales de espíritu innovador; se produce, además, mientras los ministros de Estado proceden aún de las mismas filas de la administración.

Agustín de Montiano y Luyando era de Valladolid, como la familia de su padre. Antes de 1735 se le conocía ya por su protagonismo en diversas tertulias novatoras, lo mismo en Madrid que en Palma de Mallorca, donde había residi-

43 AHN, Calatrava, Exp. $n^{\circ} .1713$; Est, Legs 3497 y $3496, \mathbf{n}^{\circ} .11$ y 12.

44 Est, Leg. 3497 y R .GÓMEZ-RIVERo, op. cit., pp.133-134.

Hispania, LIX/1, núm. 201 (1999) 193-215 
do con su tío, el regente de la Audiencia de aquella ciudad. Montiano fue, entre otras cosas, el primer director de la recién creada Academia de la Historia desde 1738. En su carrera administrativa, bastante rápida hasta su muerte en 1764 , llegó a ser Secretario de Gracia de la Cámara de Castilla. El erudito, gramático y poeta Juan de Iriarte pertenecía a una familia canaria, y había tecibido una educación muy cuidada sin necesidad de pasar por la Universidad. Durante ocho años, había estudiado latín, literatura, filosofia y matemáticas en el real colegio parisino de Clermont, dedicándose a continuación a la Historia y a las lenguas vivas. Una vez en Madrid, el confesor real le había colocado como ayudante en la Real Biblioteca en 1739; los méritos acumulados allí le valieron entrar tres años después -ya a finales de 1742- de oficial traductor en Estado, donde el sueldo y las expectativas eran mayores que en la Biblioteca 45.

José Carvajal y Lancáster, el sucesor del marqués de Villarias con la llegada de Fernando VI en 1746, señala un cambio en el origen de los ministros de Estado. Hasta finales de siglo, procederán fundamentalmente de la carrera diplomática. Dejando a un lado su familia aristocrática, Carvajal se había iniciado como colegial mayor y como magistrado, pasando por la Chancillería de Valladolid, el Consejo de Indias y el de Estado. Peto también había participado en alguna misión diplomática; y es él quien empieza a ordenar esta carrera, imbricando precisamente en ella a los oficiales del ministerio de Estado. Los secretarios de las principales embajadas-Roma, París, Viena y Londres- comenzarán a ser esos oficiales, o algunos de ellos, con Carvajal. Ricardo Wall institucionalizará después la reforma, ampliando al mismo tiempo a diez el número de oficiales del ministerio. Claro que, en esa operación, Wall conseguirá traerse de Londres al entonces secretario en aquella embajada, Bernardo del Campo, uno de los burócratas más destacados en la administración ilustrada desde los años de 1760. Se trae también a otro de esos burócratas, a Bernardo de Iriarte, sobrino del erudito canario. Es muy posible que, por lo menos a Campo, le hubiera conocido Wall en el mismo Londres, donde había sido ministro plenipotenciario, primero, y a continuación embajador, entre 1749 y 1754 .

Resulta muy dificil llegar a detectar todos, ni siquiera buena parte, de los padrinazgos que ampararon inicialmente a los empleados de los ministerios. Pero no parece arriesgado afirmar que las vías para alcanzar las plazas empiezan a cambiar con la desaparición de Felipe $V$ y del marqués de Villarias, y que toman un rumbo politicamente más definido con la muerte de Carvajal, la llegada de Wall al poder y la desaparición de Ensenada. Lo mismo en el de Estado que en otros ministerios, lo básico era entrar $y$, en segundo lugar, ir logrando algunos ascensos. A partir de ahí, el trabajo a gusto de los jefes -el ministro, posiblemente también el primero y hasta el segundo oficiales- más la capacidad de cada empleado pata destacar y para ir cteando en torno suyo una red de buenas relaciones, todo ello podía proporcionarle influencia en la colocación de otras personas y, a partir de ahí, ir haciendo más tupida su propia red de relaciones e influencias. En cualquier caso, la esta-

45 ARAH, 11/8055; y AHN, Est. Leg. 3418.

Hipania, LJX/1, núm. 201 (1999) 193-215 
bilidad en el empleo y la regularidad de los ascensos -por lentos que pudieran éstos llegar a set- son factores que limitaban el patronazgo, la plena identificación de los oficiales con los ministros y, en definitiva, la participación de los primeros en el poder político de los segundos ${ }^{4}$. A pesar de todo, en los primeros años de 1760 , el ministerio de Estado reunía ya una plantilla de empleados entre los que destacan, por sus tendencias políticas y por su espíritu ilustrado, José Nicolás de Azara, Fernando de Magallón, Bernardo del Campo, Bernardo de Iriarte, Eugenio de Llaguno y Juan de Chindurza. Es un período, sin embargo, que tebasa los límites cronológicos de este artículo y en cuya gestación, con el ministerio de Wall, no se han dado aquí más que algunas pinceladas.

46 Sobre la diferencia entre simples funcionarios y burócratas con poder políico, ver $\mathrm{M}$. Artolı en el Epílogo a R. de SANTTLLán: Memorias (1808-1856), Madrid, 1996. 